\title{
A TWO-DIMENSIONAL SINGULAR INTEGRAL EQUATION OF DIFFRACTION THEORY ${ }^{1}$
}

\author{
BY JAMES RADLOW
}

Communicated by A. Zygmund, February 28, 1964

The formulation of a problem in diffraction theory has led us to consider the two-dimensional singular integral equation

$$
\iint_{Q_{13}} f\left(t_{1}, t_{2}\right) k\left(\left|x_{1}-t_{1}\right|,\left|x_{2}-t_{2}\right|\right) d t_{1} d t_{2}=0
$$

where: $Q_{13}$ denotes the union of quadrants I, III; $f$ is unknown, but must vanish on quadrants II, IV; the equation is valid only for $x=\left(x_{1}, x_{2}\right)$ in $Q_{13}$; and $k$ is the diffraction-theoretic kernel

$$
k(x)=-(4 \pi r)^{-1} \exp (-i \beta r)
$$

with $r=+\left(x_{1}^{2}+x_{2}^{2}\right)^{1 / 2}$ and $\beta$ complex $[\operatorname{Im}(\beta)<0]$.

In earlier physical investigations, we had encountered variants of (1) in which the domains of integration and validity were (a) two contiguous quadrants (see [4]) or (b) one quadrant (see [5], [7]); and it is clear that the equation over three quadrants may be treated by methods applicable to the complementary case (b). Thus, the present study of (1) completes a theory of two-dimensional convolution-type equations with the diffraction-theoretic kernel $k$ over quadrants of the $x_{1} x_{2}$-plane. Since these equations generalize the one-dimensional convolution-type on the half-line (i.e., the classical equation of Wiener and Hopf [9]), the theory is a partial extension of Wiener and Hopf's ideas from one to two dimensions.

Our analysis may be divided into three parts:

I. Preparatory. The integral equation (1) is extended to $X$, the whole $x_{1} x_{2}$-plane, whereupon the left side becomes a convolution (Wiener's "Faltung") while the right side $h(x)$ is defined (but not known) on $X-Q_{13}$, and $h=0$ on $Q_{13}$ :

$$
f * k=h \text {. }
$$

The two-dimensional Laplace transformation ([1], Chapter VI of $[2]$, or [3]) then maps (3) into the transform equation (capital letters denote transforms; $w=\left(w_{1}, w_{2}\right)$ denotes a point in a product-space

1 This work was supported in part by the Office of Naval Research, under Contract No. Nonr 3360 (01). 
of two complex variables, with $w_{j}=u_{j}+i v_{j}$ and $j=1,2$ here and in what follows):

$$
F(w) K(w)=H(w)
$$

which is to be solved for the two unknown functions $F, H$.

It is known that $K(w)=(i / 2)\left(w_{1}^{2}+w_{2}^{2}+\beta^{2}\right)^{-1 / 2}$; thus, $K$ is analytic for $u=\left(u_{1}, u_{2}\right)$ in a product domain $B: W_{1} \times W_{2}$, where the $W_{j}$ are vertical strips interior to $\left|u_{j}\right| \leqq|\operatorname{Im}(\beta)|$. Assume next that $F$ is a distribution (cf. [3, Proposition 4.2, p. 14]) representable as

$$
F(w)=P(w) G(w),
$$

where $P$ is a polynomial, and

$$
G(w)=\left(\mathscr{L}_{1}+\mathfrak{L}_{3}\right) g(x),
$$

the restricted Laplace transform $\mathfrak{L}_{n}$ being defined by an integral over the closed $n$th quadrant:

$$
\mathfrak{L}_{n} g=\iint_{Q_{n}} g(x) \exp (-w \cdot x) d x_{1} d x_{2}
$$

$\left(w \cdot x=w_{1} x_{1}+w_{2} x_{2}\right)$. Finally, let $g(x) \exp (-w \cdot x)$ be of bounded $L_{2}$ norm over $Q_{1}, Q_{3}$ for $u$ in the respective domains

$$
\begin{aligned}
& C_{1}: u_{j}>b_{j}>0, \\
& C_{3}: u_{j}<-b_{j}<0
\end{aligned}
$$

with $C_{1} \cap C_{3}$ empty, as indicated in (8.1), (8.2), while $C_{1} \cap B$ and $C_{3} \cap B$ are nonempty.

[Remark. If $C_{1} \cap C_{3}$ is nonempty, $G \equiv 0$. The same situation is noted in [8], whose subject is the presently relevant one of characterizing two-variable Laplace transforms of functions with support in $Q_{13}$. Some of the considerations which arise are exemplified in the proof of Lemma II.2 below.] It may then be shown that:

Statement I.1. $F(w)$ and $H(w)$ as well as $K(w)$ are analytic for $u$ in $B$.

Statement I.2. $F(w)=F_{1}(w)+F_{3}(w), H(w)=H_{2}(w)+H_{4}(w)$, where: subscripts $n(n=1,2,3,4)$ denote functions analytic for $u$ in $(B, n)$, while $(B, n)$ signifies the convex closure of $B$ and the $n$th quadrant of the $u$-plane.

II. Factorization is the key step as in [9], but the single factorization lemma of Wiener and Hopf's one-dimensional theory must now be replaced by two lemmas:

LEMma II.1. $K(w)$ may be uniquely expressed as the product of four 
functions $M_{n}(w)$, analytic and nonzero for $u$ in $(B, n)$. [This is shown, and the $M_{n}(w)$ are explicitly calculated, in $\left.[5, \S 5].\right]$

Lemma II.2. $K_{13}(w)=M_{1}(w) M_{3}(w)$ and $K_{24}(w)=M_{2}(w) M_{4}(w)$ are analytic and nonzero in the respective pairs of disjoint u-domains $\left(-\infty<v_{j}<+\infty\right.$ throughout) $q_{1}, q_{3}$ and $q_{2}, q_{4}$, where we have (for any $\delta>0)$

$$
q_{1}:\left(u_{1}+u_{2}\right) \geqq(|\operatorname{Im}(\beta)|+\delta) \quad\left(u_{j}>0\right)
$$

and $q_{2}, q_{3}, q_{4}$ are successive reflections of $q_{1}$ in $u_{2}>0, u_{1}<0, u_{2}<0$.

Proof. Introduce the function

$$
\begin{aligned}
\phi(x) & =N_{0}(\beta r), & & x \in Q_{13} \\
& =0, & & x \in Q_{24}
\end{aligned}
$$

where $N_{0}(\beta r)$ is the Bessel function of the second kind (Neumann's function). The image of $\phi$ under two-dimensional Laplace transformation is, as shown in [6],

$$
\Phi(w)=2 i\left(w_{1}^{2}+w_{2}^{2}+\beta^{2}\right)^{-1}\left[-i+w_{1} s_{2} S_{2}+w_{2} s_{1} S_{1}\right],
$$

with

$$
\begin{aligned}
s_{j} & =\left(w_{j}^{2}+\beta^{2}\right)^{-1 / 2} \quad\left(s_{j}=\beta^{-1} \text { at } w_{j}=0\right) \\
S_{j} & =\left(2 i \pi^{-1}\right) \log \left[\beta^{-1}\left(w_{j}+s_{j}^{-1}\right)\right]
\end{aligned}
$$

and, significantly,

$$
\mathfrak{L} \phi(x)=2 \mathfrak{L}_{1} \phi(x)=2 \mathfrak{L}_{3} \phi(x) .
$$

As appears from (12), $\Phi(w) \equiv £ \phi(x)$ is analytic for $u$ in $q_{1}$ and for $u$ in $q_{3}$. The same is true of $K_{13}(w)$, since it may be shown (by the reasoning of $[5, \S 5])$ that

$$
K_{13}(w)=\exp \left[-\int^{\beta} \Phi(w ; \beta) d \beta\right]
$$

where $\Phi$ is written $\Phi(w ; \beta)$ to emphasize the dependence on $\beta$, and it is understood that $\beta$ is allowed to vary in a small neighborhood of its fixed value for purposes of the integration. The assertion for $K_{13}$ is therefore proved, and the proof for $K_{24}$ follows by symmetry.

III. Solutions of the transform equation and the integral equation. Two theorems may now be proved without difficulty (the first requires only verification): 
ThEOREM III.1. The transform equation (4) has the solutions

$$
\begin{aligned}
& F(w)=c_{0}\left[K_{13}(w)\right]^{-1}, \\
& H(w)=c_{0} K_{24}(w)
\end{aligned}
$$

where $c_{0}$ is an arbitrary constant [the same arbitrary constant in (14.1), (14.2)].

Theorem III.2. The functions $F, H$ of (14.1), (14.2) possess twovariable Laplace inverses $f(x), h(x)$, and the latter pair are literal solutions of (3). [The function $f(x)$ is of course a literal solution of (1), as well as of the extended equation (3).]

\section{REFERENCES}

1. S. Bochner, Bounded analytic functions in several variables and multiple Laplace integrals, Amer. J. Math. 59 (1937), 732-738.

2. S. Bochner and W. T. Martin, Several complex variables, Princeton Univ. Press, Princeton, N. J., 1948.

3. J. Leray, Hyperbolic differential equations, lecture notes, Inst. for Advanced Study, Princeton, N. J., 1954.

4. J. Radlow, Diffraction of a dipole field by a unidirectionally conducting semiinfinite screen, Quart. Appl. Math. 17 (1959), 113-127.

5. - Diffraction by a quarter-plane, Arch. Rational Mech. Anal. 8 (1961), $139-158$.

6. - On the double Laplace transforms of some Green's functions, J. Math. and Phys. 38 (1959), 203-205.

7. - Diffraction by a right-angled dielectric wedge, Internat. J. Eng. Sci. (to appear).

8. - A two-dimensional version of Laplace's integral equation, Abstract 603-141, Notices Amer. Math. Soc. 10 (1963), 474.

9. N. Wiener and E. Hopf, Über eine Klasse Singulärer Integralgleichungen, S.-B. Berlin Akad. Wiss. (1931), 696-706.

Purdue University 